\title{
Dose correction in lung for HDR breast brachytherapy
}

\author{
Eric Slessinger, MS', Eric Pepin, PhD², Qingya Zhao, PhD', Li Zhao, PhD², Indra Das, PhD'.3 \\ Indiana University Simon Cancer Center, Indiana University School of Medicine, Indiana, 2Purdue University. West Lafayette, Indiana, \\ Indiana University, Health Proton Therapy Center, Bloomington, Indiana, USA
}

\begin{abstract}
Purpose: To evaluate the dosimetric impact of lung tissue in Ir-192 APBI.

Material and methods: In a $40 \times 40 \times 40 \mathrm{~cm}^{3}$ water tank, an Accelerated Partial Breast Irradiation (APBI) brachytherapy balloon inflated to $4 \mathrm{~cm}$ diameter was situated directly below the center of a $30 \times 30 \times 1 \mathrm{~cm}^{3}$ solid water slab. Nine $\mathrm{cm}$ of solid water was stacked above the $1 \mathrm{~cm}$ base. A parallel plate ion chamber was centered above the base and ionization current measurements were taken from the central HDR source dwell position for channels 1, 2, 3 and 5 of the balloon. Additional ionization data was acquired in the $9 \mathrm{~cm}$ stack at $1 \mathrm{~cm}$ increments. A comparable data set was also measured after replacing the $9 \mathrm{~cm}$ solid water stack with cork slabs. The ratios of measurements in the two phantoms were calculated and compared to predicted results of a commercial treatment planning system.

Results: Lower dose was measured in the cork within $1 \mathrm{~cm}$ of the cork/solid water interface possibly due to backscatter effects. Higher dose was measured beyond $1 \mathrm{~cm}$ from the cork/solid water interface, increasing with path length up to $15 \%$ at $9 \mathrm{~cm}$ depth in cork. The treatment planning system did not predict either dose effect.

Conclusions: This study investigates the dosimetry of low density material when the breast is treated with Ir-192 brachytherapy. HDR dose from Ir-192 in a cork media is shown to be significantly different than in unit density media. These dose differences are not predicted in most commercial brachytherapy planning systems. Empirical models based on measurements could be used to estimate lung dose associated with HDR breast brachytherapy.

J Contemp Brachyther 2012; 4, 2: 106-110 DOI: $10.5114 /$ jcb.2012.29367
\end{abstract}

Key words: lung, dosimetry, partial breast irradiation, HDR.

\section{Purpose}

Accelerated Partial Breast Irradiation (APBI) using Iridium-192 (Ir-192) in high dose rate (HDR) brachytherapy is becoming a common radiation oncology treatment modality for breast conserving therapy [1-5]. APBI is delivered in a hypofractionated regimen that treats a $1 \mathrm{~cm}$ margin around the resected tumor cavity, reducing the dose to normal tissue when compared to three dimensional (3D) conformal external beam radiotherapy (EBRT) [6-9]. APBI has been shown to have good cosmetic outcomes while providing a level of convenience that makes adjuvant radiation therapy accessible to more patients with breast cancer [10-12]. Proximity of the planning target volume (PTV) to the lung makes lung dose evaluation and PTV dose accuracy important. However, reporting an accurate lung dose is a dosimetric challenge due to the paucity of commercial brachytherapy treatment planning systems (TPS) capable of dose calculation in heterogeneous media [4,13-16]. Most TPS calculation algorithms conforming to TG-43 recommendations are based on Monte-Carlo simulations of a single source in a $15 \mathrm{~cm}$ water phantom $[13,16-20]$. As such, they do not account for differences in tissue size, shape or density [16].
One of several devices used in APBI is the Contura Multi-Lumen Balloon (CMLB) (SenoRx, Inc., Irvine, CA, USA). The CMLB is surgically inserted into the resulting cavity following tumor resection; the CMLB is then filled with saline and radiographically opaque contrast solution, for localization during treatment planning and for monitoring the diameter prior to each treatment. Within the balloon are five channels into which an Ir-192 source can be driven by a remote afterloader. It has been shown that the versatility afforded by the multiple channels may offer reduced skin and rib doses, greater compliance with treatment planning goals and no increase in toxicity when compared to single channel devices $[5,21,22]$. Additionally, the CMLB has a vacuum port through which fluid and air can be removed from the treatment cavity to optimize target conformity, and which otherwise might introduce further heterogeneities [23]. However, it has also been shown that dose perturbations can be introduced through the use of certain contrast solutions such as Iodine $[3,4,24,25]$.

The lungs are recognized as organs at risk (OAR) in partial breast radiotherapy [26-28]. For 3D EBRT, the NSABP trial B-39 requires that not more than $15 \%$ of the ipsilateral lung receive $30 \%$ of the prescribed dose, and specifies that 
the dose computations should take into account tissue density. The NSABP trial B-39 is a randomized phase III study of conventional whole breast irradiation (WBI) versus partial breast irradiation (PBI) for women with Stage 0, I or II breast cancer. Lung dose has been reported to be lower from brachytherapy than from 3D EBRT $[13,29]$. However, lung dose comparisons for breast EBRT and brachytherapy are questionable if tissue density is not accurately accounted for in dose calculations. Inconsistencies arise since most commercial brachytherapy TPS do not take into account the effect of tissue densities while EBRT TPS are required to. Despite that, many studies report lung dose from breast brachytherapy $[26,28,29]$. It has been reported that the inverse square fall-off is an appropriate approximation because scatter buildup offsets attenuation; it has also been reported that there are minimal heterogeneity effects when using Ir-192 [15]. However, several Monte-Carlo studies have demonstrated measurable heterogeneity effects during brachytherapy near the lung [4,14-16]. These findings complement studies showing that the lung-soft tissue interface is the region most dependent on dose calculation algorithm and that these differences can be statistically significant [30-32]. This study attempts to address the issue of lung dose from HDR breast brachytherapy, enabling valid dose comparisons between EBRT and brachytherapy. In it, a CMLB device was used to make dosimetric comparisons of measurements in heterogeneous and homogeneous phantoms. TPS calculations of dose in the two phantoms were also compared. The results of this experiment are intended to improve lung dose estimation for partial breast brachytherapy.

\section{Material and methods}

The CMLB brachytherapy device for APBI is a spherical balloon filled with saline and contrast, containing five channels into which an HDR Ir-192 source can be driven (Fig. 1). A MicroSelectron V2 HDR (Nucletron B.V., Veenen-

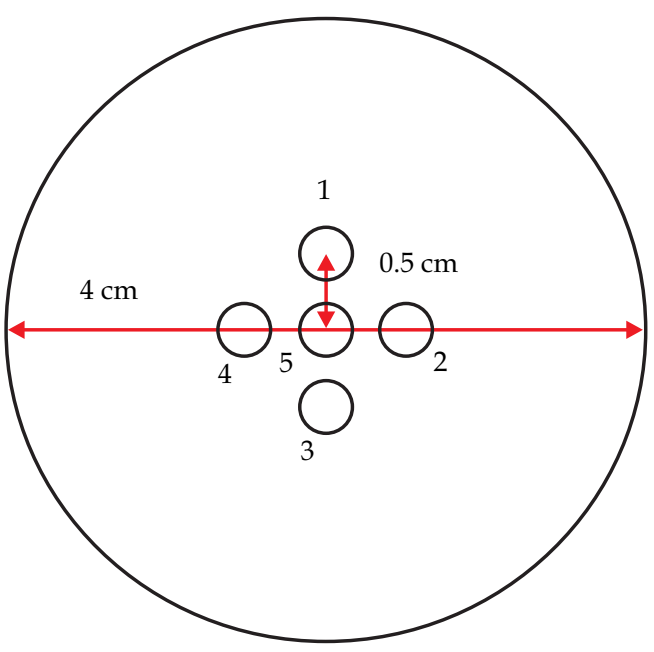

Fig. 1. Cross-sectional diagram of the Contura through channel centers daal, The Netherlands) was used to control the Ir-192 source. The CMLB was filled with $37 \mathrm{~cm}^{3}$ of saline to $4 \mathrm{~cm}$ diameter and positioned in a $40 \times 40 \times 40 \mathrm{~cm}^{3}$ water tank beneath a $30 \times 30 \times 1 \mathrm{~cm}^{3}$ solid water slab. Solid water (Model Solid Water, RMI Inc, Madison, WI, USA) is a convenient water substitute material for phantom dosimetry, composed of epoxy resins and powder with density $1.04 \mathrm{gm} / \mathrm{cm}^{2}$. A Scanditronix parallel plate ion chamber (Type NACP-02, IBA Dosimetry America, Inc., Bartlett, TN, USA) was centered within a $30 \times 30 \times 1 \mathrm{~cm}^{3}$ solid water slab, which was placed on the initial slab. The ion chamber was aligned to channel 1 of the CMLB with the incident chamber surface facing the CMLB (Fig. 2). Additional $30 \times 30 \mathrm{~cm}$ solid water slabs of varying thickness were added to the stack for a total height of $10 \mathrm{~cm}$. A CNMC K602 electrometer (CNMC Company Inc., Nashville, TN, USA) was used to measure the ionization current. The 'sweet spot' dwell positions for channels 1, 2, 3 and 5 of the CMLB were established through an iterative process at the source position that gave the highest ionization reading. Channel 4 was not included in the study, because it is represented by channel 2. Ionization measurements were taken at $1 \mathrm{~cm}$ increments within the solid water stack, up to $6 \mathrm{~cm}$ from the CMLB surface, using the established 'sweet spot' dwell position for each channel (Fig. 3A). The measurements were repeated after replacing the top $9 \mathrm{~cm}$ of solid water with $30 \times 30 \times 1.27 \mathrm{~cm}$ compact cork slabs $\left(\rho=0.25 \mathrm{~g} / \mathrm{cm}^{3}\right)$ (Fig. 3B). Cork is a common substitute material for lung in dosimetry studies [33-39]. The measured ionization currents were normalized for source activity, temperature and atmospheric pressure.

The cork data were acquired at different depth increments than solid water due to differing slab thicknesses. Therefore, the data were fitted based on depth interpolation before being compared to the solid water data. The data were fitted using power functions of the form:

$$
f(x)=\alpha \cdot x^{-\beta}
$$

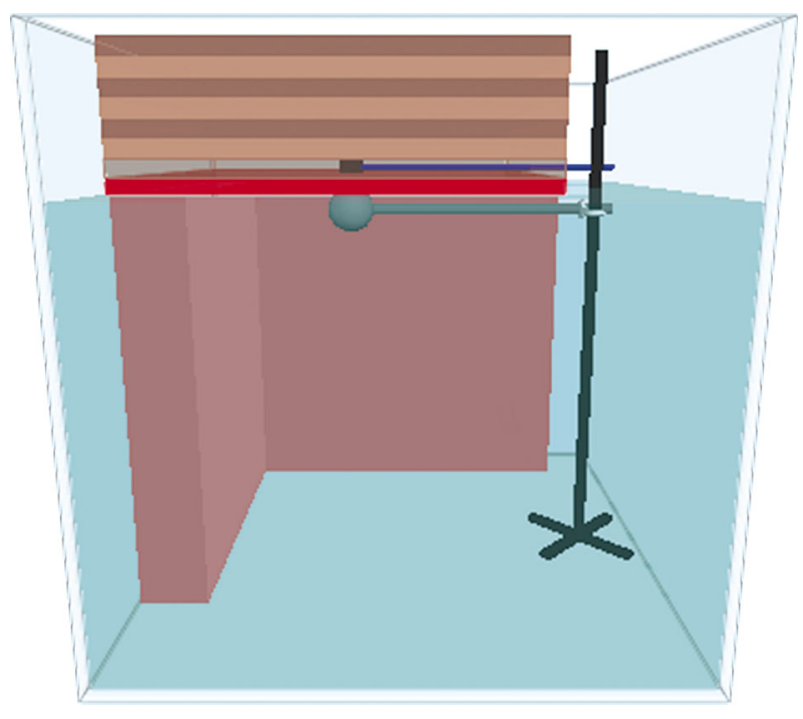

Fig. 2. Experimental setup in water tank showing the ion chamber above Contura 
A

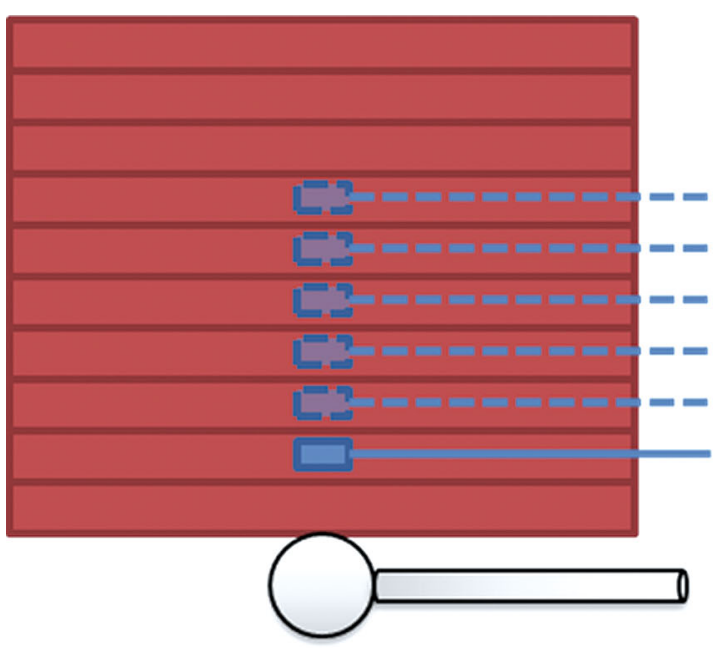

B

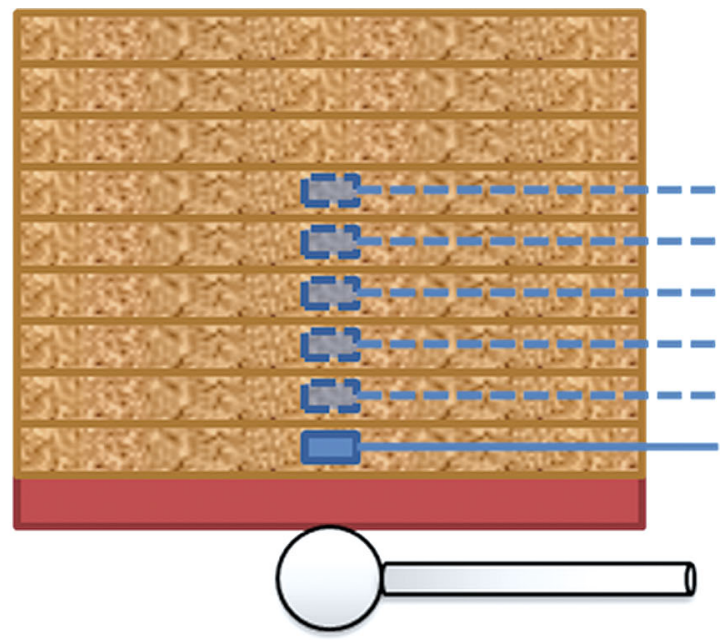

Fig. 3. Setup for measurements in (A) solid water phantom and (B) cork phantom

where $\alpha$ and $\beta$ are fitting parameters. Data analysis utilized the application Microsoft Excel, Version 10 (Microsoft, Inc, Redmond, WA, USA). Resultant data were normalized to the $1 \mathrm{~cm}$ depth in solid water that was common to both setups. Ratios of the fitted cork to solid water measurements were calculated. The data were plotted as relative dose vs. source-to-chamber distance. Measurement data were repeatedly acquired in two experimental sessions and average values analyzed. The two experimental phantom setups were also CT scanned to compute doses using the Oncentra MasterPlan treatment planning system (Nucletron B.V.). Dose computations were evaluated at points corresponding to the measured dose point range from channel one.

\section{Results}

The acquired data were fitted to eq. 1 with $R^{2}$ values $>0.99$. Dose ratios were calculated by considering the analytic ratios of the trend line equations for each channel and are shown in Fig. 4. This analytic result agreed with interpolated ratios at the measured distances.

The first data point of each channel was measured with identical attenuation, $1 \mathrm{~cm}$ of solid water, but with different backscatter conditions. At this first point, averaged over all channels, there was 3.6\% $(\sigma=2.7 \%)$ less dose in the cork phantom than in the solid water phantom. Over the measurement range (6 $\mathrm{cm}$ in cork), the dose in cork exceeds the dose in solid water by as much as $10 \%$. The magnitude of this dose effect increases with path length. Table 1 lists the computed dose comparisons for both phantoms. Despite the difference in phantom composition, the dose computation results agree to $0.5 \pm 0.005 \%$. The dose calculations using the Oncentra MasterPlan brachytherapy planning system do not predict either the backscatter effect or the increase in dose with path length through cork (Table 1).

\section{Discussion}

Lung is an organ at risk (OAR) in breast radiotherapy [26-28]. For 3D EBRT, the NSABP trial B-39 requires that
$<15 \%$ of the ipsilateral lung can receive $30 \%$ of the prescribed dose and specifies that the dose computations take into account tissue density. Lung dose has been reported to be lower from brachytherapy than from external beam [13,29]. However, lung dose comparisons for breast teletherapy and brachytherapy are questionable if tissue density is not determined consistently. That is clearly the case since most brachytherapy planning systems do not take into account the effect of tissue densities while the external beam planning systems are required to. Despite that, many studies report lung dose from breast brachytherapy $[26,28,29]$. One can argue that the effects from inverse square falloff predominate, but reporting on lung dose needs some correction for valid comparison to EBRT doses. This study has attempted to address the issue of lung dose from HDR breast brachytherapy. Understanding the effect of low density tissue on breast brachytherapy dose will validate dose comparisons between teletherapy and brachytherapy. In this study, measurements indicate low doses adjacent to the interface of unit density and low density structures due to reduced backscattering in the low density material. This dose perturbation effect has also been reported based on Monte Carlo calculations [40].

The measurement results in this study also demonstrate that the dose in low density media relative to unit density increases with path length. However, the degree to which this dose increase occurs may be overstated in this study since the solid water material has been shown to attenuate by approximately $4 \%$ more than liquid water [41]. Considering that tissue densities are variable for different patients, as well as across a given structure, the results of this study should be regarded qualitatively.

\section{Conclusions}

In an experiment evaluating the dose differences between Contura based HDR brachytherapy in water and lung equivalents, measurements demonstrated significant dose differences at the inhomogeneous interface. Measurements further demonstrated that the differences increase with path 
A

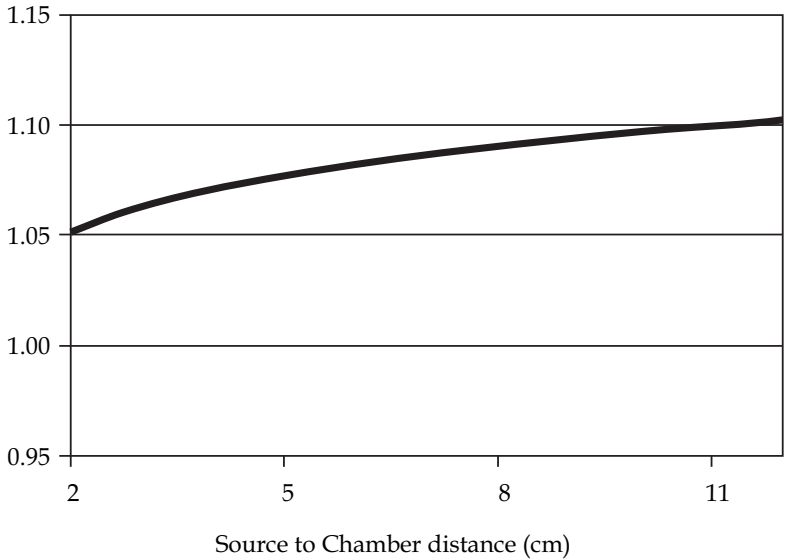

C

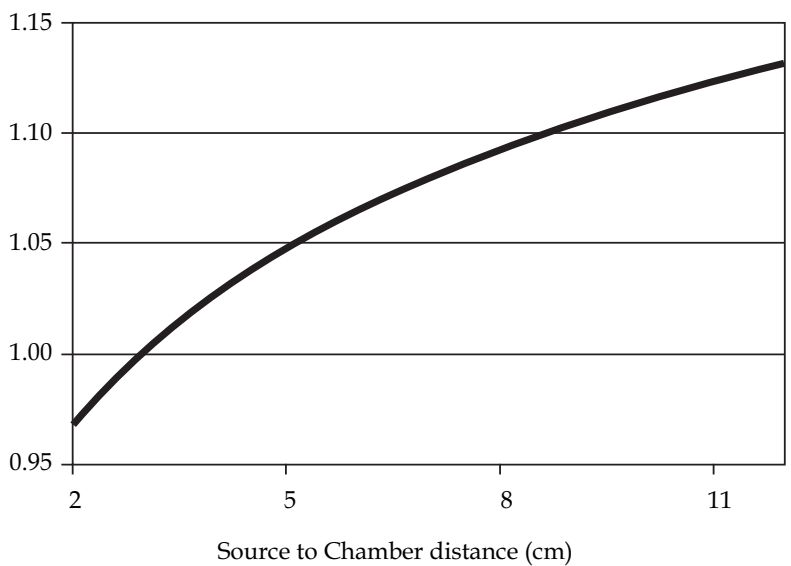

B

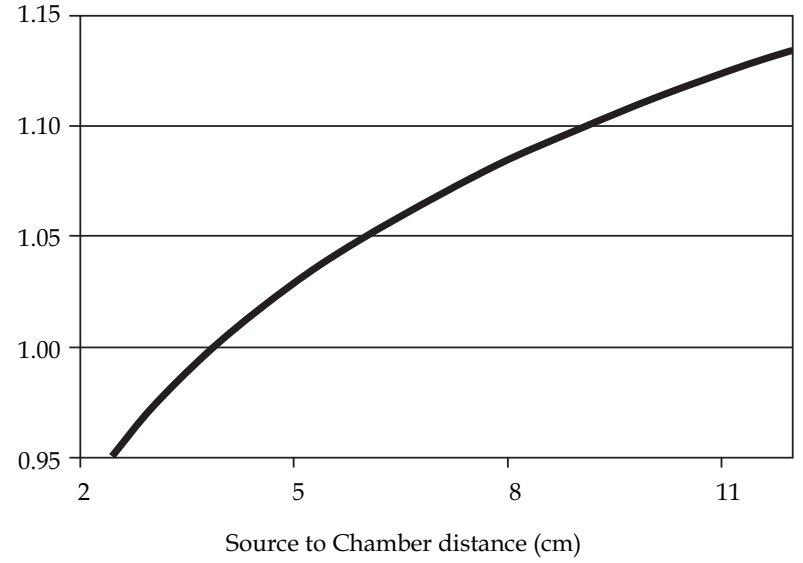

D

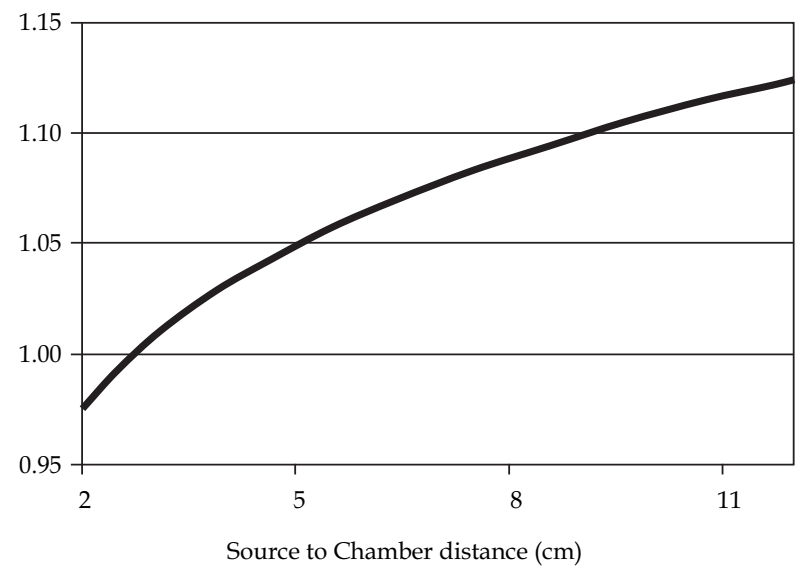

Fig. 4. Plots of the trend line-based analytical ratios of relative dose in cork to solid water vs. source-to-chamber distance for (A) Channel 1 with the cork-solid water interface at $2.5 \mathrm{~cm}$, (B) Channel 2 with the cork-solid water interface at $3.04 \mathrm{~cm}$, (C) Channel 3 with the cork-solid water interface at $3.5 \mathrm{~cm}$, and (D) Channel 5 with the cork-solid water interface at $3 \mathrm{~cm}$

Table 1. Comparison of Oncentra dose calculations from channel one for the two phantom configurations

\begin{tabular}{lcccc} 
Solid Water & \multicolumn{2}{c}{ Cork } & Ratio \\
\hline Depth [mm] & Relative Dose & Depth [mm] & Relative Dose & Cork/Solid Water \\
\hline 30 & 58.78 & 30 & 57.99 & 0.99 \\
\hline 40 & 32.09 & 40 & 31.79 & 0.99 \\
\hline 50 & 20.07 & 50 & 19.94 & 0.99 \\
\hline 60 & 13.67 & 60 & 13.62 & 1.00 \\
\hline 80 & 7.47 & 80 & 7.47 & 1.00 \\
\hline 100 & 4.67 & 100 & 4.68 & 1.00
\end{tabular}

length through the low density medium. These findings are not predicted by most commercial brachytherapy planning systems and may serve as a model to better understand lung dose during breast brachytherapy with Ir-192, enabling proper dose reporting for OAR.

\section{References}

1. Kim Y, Werts ED, Trombetta MG et al. Evaluation of the interfractional biological effective dose (BED) variation in MammoSite high dose rate brachytherapy. J Appl Clin Med Phys 2010; 11: 3228
2. Dragun AE, Harper JL, Olyejar SE et al. The use of adjuvant high-dose-rate breast brachytherapy in patients with collagen vascular disease: a collaborative experience. Brachytherapy 2011; 10: 121-127.

3. Strauss JB, Dickler A. Accelerated partial breast irradiation utilizing balloon brachytherapy techniques. Radiother Oncol 2009; 91: 157-165.

4. Richardson SL, Pino R. Dosimetric effects of an air cavity for the SAVITM partial breast irradiation applicator. Med Phys 2010; 37: 3919-3926.

5. Cuttino LW, Todor D, Rosu M et al. A comparison of skin and chest wall dose delivered with multicatheter, Contura multi- 
lumen balloon, and MammoSite breast brachytherapy. Int J Radiat Oncol Biol Phys 2011; 79: 34-38.

6. Major T, Polgar C, Mangel L et al. CT based conformal brachytherapy treatment planning. Magy Onkol 2000; 44: 109-115.

7. Njeh CF, Saunders MW, Langton CM. Accelerated Partial Breast Irradiation (APBI): a review of available techniques. Radiat Oncol 2010; 5: 90.

8. Yarnold J, Bentzen SM, Coles C et al. Hypofractionated wholebreast radiotherapy for women with early breast cancer: myths and realities. Int J Radiat Oncol Biol Phys 2011; 79: 1-9.

9. Whelan TJ, Pignol JP, Levine MN et al. Long-term results of hypofractionated radiation therapy for breast cancer. $N$ Engl J Med 2010; 362: 513-520.

10. Wazer D, Kaufman S, Cuttino L et al. Accelerated partial breast irradiation: an analysis of variables associated with late toxicity and long-term cosmetic outcome after high-dose-rate interstitial brachytherapy. Int J Radiat Oncol Biol Phys 2006; 64: 489-495.

11. Rose C, Recht A. Accelerated partial-breast irradiation (APBI): let's give it a good test. Int J Radiat Oncol Biol Phys 2003; 57: 1217-1218.

12. Keisch M, Vicini F, Beitsch P et al. American Society of Breast Surgeons MammoSite Radiation Therapy System Registry Trial: ductal carcinoma-in-situ subset analysis - 4-year data in 194 treated lesions. Am J Surg 2009; 198: 505-507.

13. Rivard MJ, Venselaar JL, Beaulieu L. The evolution of brachytherapy treatment planning. Med Phys 2009; 36: 2136-2153.

14. Zhang Z, Parsai EI, Feldmeier JJ. Three-dimensional quantitative dose reduction analysis in MammoSite balloon by Monte Carlo calculations. J Appl Clin Med Phys 2007; 8: 2669.

15. Poon E, Verhaegen F. A CT-based analytical dose calculation method for HDR ${ }^{192}$ Ir brachytherapy. Med Phys 2009; 36: 3982.

16. Anagnostopoulos G, Baltas D, Karaiskos P et al. An analytical dosimetry model as a step towards accounting for inhomogeneities and bounded geometries in 192Ir brachytherapy treatment planning. Phys Med Biol 2003; 48: 1625-1647.

17. Williamson JF, Li Z. Monte Carlo aided dosimetry of the microselectron pulsed and high dose-rate 192Ir sources. Med Phys 1995; 22: 809-819.

18. Daskalov GM, Loffler E, Williamson JF. Monte Carlo-aided dosimetry of a new high dose-rate brachytherapy source. Med Phys 1998; 25: 2200-2208.

19. Karaiskos P, Angelopoulos A, Baras P et al. A Monte Carlo investigation of the dosimetric characteristics of the VariSource 192Ir high dose rate brachytherapy source. Med Phys 1999; 26: 1498-1502.

20. Nath R, Anderson LL, Luxton G et al. Dosimetry of interstitial brachytherapy sources: recommendations of the AAPM Radiation Therapy Committee Task Group No. 43. American Association of Physicists in Medicine. Med Phys 1995; 22: 209-234.

21. Brown S, McLaughlin M, Pope DK et al. A dosimetric comparison of the Contura multilumen balloon breast brachytherapy catheter vs. the single-lumen MammoSite balloon device in patients treated with accelerated partial breast irradiation at a single institution. Brachytherapy 2011; 10: 68-73.

22. Wilder RB, Curcio LD, Khanijou RK et al. A Contura catheter offers dosimetric advantages over a MammoSite catheter that increase the applicability of accelerated partial breast irradiation. Brachytherapy 2009; 8: 373-378.

23. Tokita KM, Cuttino LW, Vicini FA et al. Optimal application of the Contura multilumen balloon breast brachytherapy catheter vacuum port to deliver accelerated partial breast irradiation. Brachytherapy 2011; 10: 184-189.

24. Kassas B, Mourtada F, Horton JL et al. Contrast effects on dosimetry of a partial breast irradiation system. Med Phys 2004; 31: 1976-1979.
25. Kirk MC, Hsi WC, Chu JC et al. Dose perturbation induced by radiographic contrast inside brachytherapy balloon applicators. Med Phys 2004; 31: 1219-1224.

26. Garza R, Albuquerque K, Sethi A. Lung and cardiac tissue doses in left breast cancer patients treated with single-source breast brachytherapy compared to external beam tangent fields. Brachytherapy 2006; 5: 235-238.

27. Scanderbeg D, Yashar C, White G et al. Evaluation of three APBI techniques under NSABP B-39 guidelines. J Appl Clin Med Phys 2010; 11: 3021.

28. Yashar CM, Blair S, Wallace A et al. Initial clinical experience with the Strut-Adjusted Volume Implant brachytherapy applicator for accelerated partial breast irradiation. Brachytherapy 2009; 8: 367-372.

29. Berger D, Kauer-Dorner D, Seitz W et al. Concepts for critical organ dosimetry in three-dimensional image-based breast brachytherapy. Brachytherapy 2008; 7: 320-326.

30. Xiao Y, Papiez L, Paulus R et al. Dosimetric evaluation of heterogeneity corrections for RTOG 0236: stereotactic body radiotherapy of inoperable stage I-II non-small-cell lung cancer. Int J Radiat Oncol Biol Phys 2009; 73: 1235-1242.

31. Carrasco P, Jornet N, Duch MA et al. Comparison of dose calculation algorithms in phantoms with lung equivalent heterogeneities under conditions of lateral electronic disequilibrium. Med Phys 2004; 31: 2899.

32. Morgan A, Knoos T, McNee S et al. Clinical implications of the implementation of advanced treatment planning algorithms for thoracic treatments. Radiother Oncol 2008; 86: 48-54.

33. Bragg CM, Conway J. Dosimetric verification of the anisotropic analytical algorithm for radiotherapy treatment planning. Radiother Oncol 2006; 81: 315-323.

34. Chang KP, Hung SH, Chie YH et al. A comparison of physical and dosimetric properties of lung substitute materials. Med Phys 2012; 39: 2013.

35. Kohda E, Shigematsu N. Measurement of lung density by computed tomography: implication for radiotherapy. Keio J Med 1989; 38: 454-463.

36. Mesbahi A, Allahvedi M, Gheraati H et al. Experimental evaluation of ALFARD treatment planning system for 6MV photon irradiation: a lung case study. Rep Pract Oncol Radiother 2004; 9: 217-221.

37. Osei EK, Darko J, Mosseri A et al. EGSNRC Monte Carlo study of the effect of photon energy and field margin in phantoms simulating small lung lesions. Med Phys 2003; 30: 2706.

38. Uniyal SC, Sharma SD, Naithani UC. Dosimetric verification of a high dose rate brachytherapy treatment planning system in homogeneous and heterogeneous media. Physica Medica 2012; Epub ahead of print.

39. Van Dyk J, Keane TJ, Rider WD. Lung density as measured by computerized tomography: implications for radiotherapy. Int J Radiat Oncol Biol Phys 1982; 8: 1353-1372.

40. Pantelis E, Papagiannis P, Karaiskos P et al. The effect of finite patient dimensions and tissue inhomogeneities on dosimetry planning of 192Ir HDR breast brachytherapy: a Monte Carlo dose verification study. Int J Radiat Oncol Biol Phys 2005; 61: 1596-1602.

41. Tailor R, Tolani N, Ibbott GS. Thermoluminescence dosimetry measurements of brachytherapy sources in liquid water. Med Phys 2008; 35: 4063-4069. 